\title{
Flow Structures Inside a Rotor-Stator Cavity
}

\author{
T. GEIS*, J. EBNER, S. KIM and S. WITTIG
}

Lehrstuhl und Institut für Thermische Strömungsmaschinen, University of Karlsruhe (T.H.), 76128 Karlsruhe, Germany

(Received 2 May 2000; In final form 31 May 2000)

\begin{abstract}
This paper describes an experimental investigation initiated to determine the threedimensional flow field inside the rim seal cavity of a double-shrouded rotor-stator system. Thereby, the effects caused by perturbances in the rotor wall were additionally examined. The objective of this work is to provide detailed information about the mechanisms that can promote elevated temperature levels in the high pressure section of a gas turbine. Both ingested hot gas and windage heating generated at the rotor-stator interface can severely affect the material temperatures and thus considerably increase the thermal load of the rotating parts.

The flow velocities were measured by means of an advanced LDV system capable of providing phase-resolved data. The flow field was determined for two different rotorstator combinations. One of the rotor disks contained small rectangular cavities, located at the disk rim and arranged uniformly in the circumferential direction. These eiements are referred to as the shank cavities of the rotor disk.

The mechanical torque was measured to demonstrate the influence of these elements on the windage power. The measurements were performed at operating conditions that are typical for aero-engines. It is shown that a perturbed rotor surface can raise the drag notably. The experiments were conducted in a high speed test rig at rotational Reynolds numbers up to $\operatorname{Re}_{\varphi} \approx 4.2 * 10^{6}$. The data were plotted as the dimensionless moment coefficient $c_{M}$ and correlated with $\operatorname{Re}_{\varphi}$ and the dimensionless cooling flow rate $c_{w}$.
\end{abstract}

Keywords: Internal air system; LDV; Moment coefficient; Rotor-stator system; Separated flow

\section{INTRODUCTION}

The thermal management of a gas turbine high pressure section is receiving considerable attention. A sufficient amount of cooling air must be provided to protect those parts that are exposed to a more and more hostile environment. Due to the aspired improvement of the overall engine efficiency however, cooling air must further be limited and hence, efforts must be focussed on a more effective cooling methodology until more resistant construction materials are found. In terms of the rotor-stator interface, a better design will be characterised by a minimised amount of coolant

\footnotetext{
* Address for correspondence: Lehrstuhl und Institut für Thermische Strömungsmaschinen, Universität Karlsruhe, Kaiserstrasse 12, 76128 Karlsruhe, Germany. Tel.: +49-721-608-6016, Fax: +49-721-699222, e-mail: torsten.geis@its.uni-karlsruhe.de
} 
necessary to remove the heat successfully from the rotor disk. It will also require a minimum amount of sealing air to prevent excessive ingress from the hot gas path. Last but not least, windage heating and the power losses generated at the interface will be low in such an optimised rotor-stator system.

A comprehensive study was conducted by Pincombe (1989) to investigate the flow structures inside rotating cavities, rotating tubes and rotorstator systems. For various operating conditions, the author applied a combined flow visualisation and LDA technique to present qualitative and quantitative pictures of the existing flow field. Since the work was focussed on a general understanding of the basic nature of the flow, two-dimensional, axisymmetric geometries were considered.

Owen and Rogers (1989) performed an elaborate study that covers the subjects heat transfer, windage heating, hot gas ingestion and flow. A variation of the rotor geometry was focussed on different seal configurations mainly to show the influence on the sealing performance.

Nece and Daily (1960) used grit papers cemented to the rotor disk surfaces in order to investigate the effects of surface roughness on the frictional resistance. For the turbulent flow regime they found that the resistance increases with decreasing grit size. In terms of a large scale roughness, data is provided by Gärtner (1998) and Zimmermann et al. (1986) for the case where bolts are present on the rotor disk.

Applying results from simplified models to more complex and realistic, three-dimensional geometries is critical in most of the cases. Owing to a lack of data, the impact of a modified rotor-stator geometry on the fluid dynamics can hardly be predicted with the desired accuracy. Employing CFD is still unsatisfactory unless benchmark experiments are available to validate the numerical data.

To the authors' knowledge, no detailed experimental data has been published for rectangular cavities in gas turbine rotor disks. These types of perturbances are typically found in the vicinity of the shroud and referred to as the shank cavities.
Research related to the present problem was undertaken for the non-rotational case only. The so called ' $d$ ' type roughness typified by depressions or narrow grooves in a wall and its associated flow pattern is described by Perry et al. (1969). Similar work regarding the turbulent separated flow and the momentum transfer mechanisms associated with these elements was performed by Kistler and Tan (1967) and Haugen and Dhanak (1966). It is indicated by the authors that the drag force acting on the 'roughness' elements is influenced by the shape of the cavity. The depth of these elements in turn can provoke violent fluctuations of the pressure distribution in and near the cavity, as reported from Roshko (1955). These erratic and intermittent variations are due to the unstable character of the vortex mechanism inside the groove.

The present study aims to gain a more detailed understanding of the flow mechanisms associated with cutouts in a rotating disk. While the subject of hot gas ingestion was earlier addressed by the authors (Geis et al., 1999), the present work is focussed on the power losses associated with these elements. By producing quantitative results for the flow field and the moment coefficient, the existing data base can be extended. However, in terms of further changes in the geometry, the work is far from being complete. There is considerable scope for future investigations.

\section{EXPERIMENTAL APPARATUS}

\section{Test Section}

The test section employed in the present study is shown in Figure 1. While all stationary parts of this simple rotor-stator system are made from steel, the rotor disk is made from an aluminum7075 alloy strong enough to withstand the applied loads. The disk being $496 \mathrm{~mm}$ in diameter was accelerated to a maximum speed of $10000 \mathrm{rpm}$. Hence, rotational Reynolds numbers up to $4.2 \times 10^{6}$ were attained. The rotor-stator system 

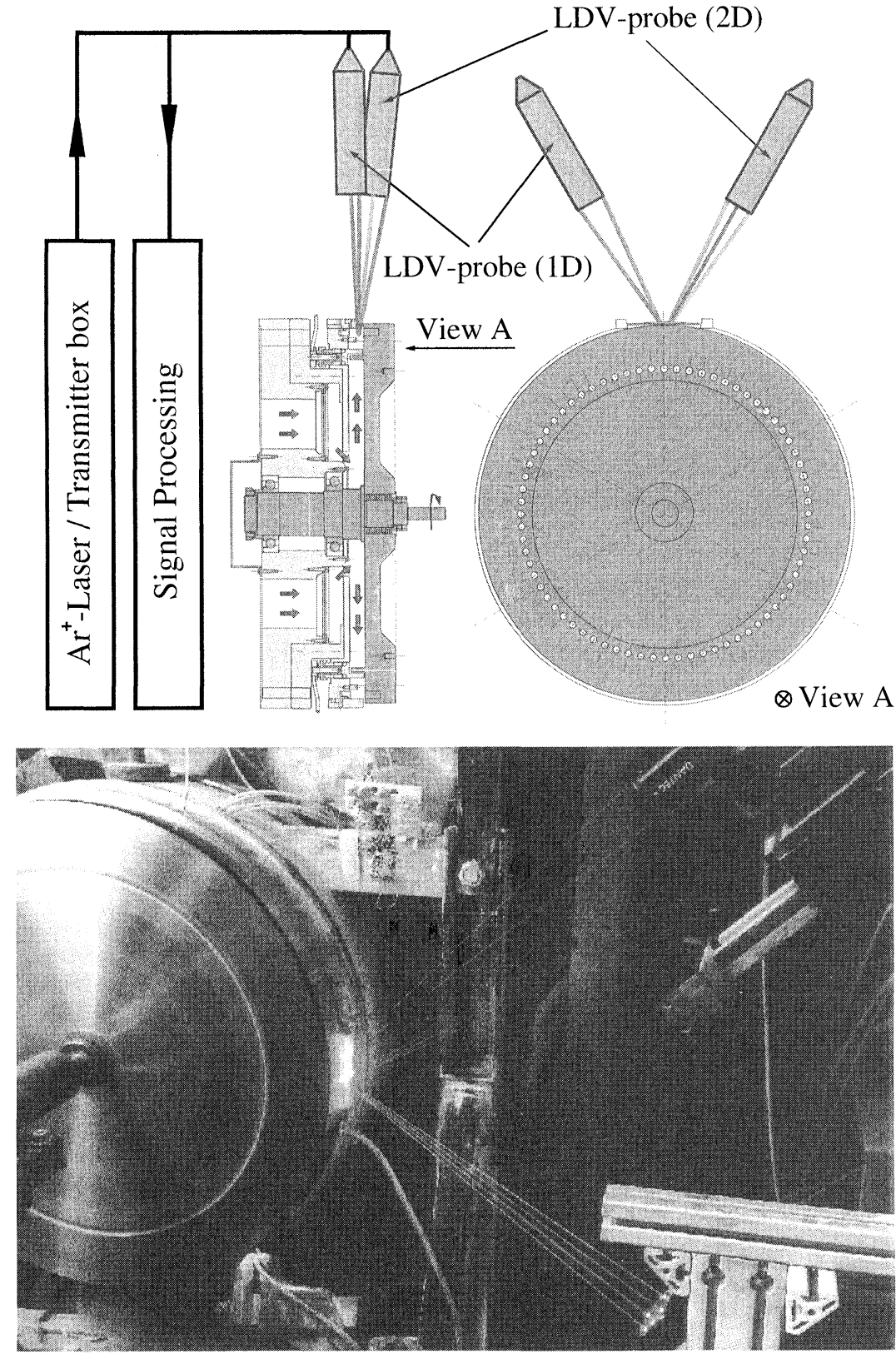

FIGURE 1 LDV-setup.

consists of one axial seal at the outer shroud and a combined axial and radial seal that divides the space between the disk and the stator into an outer and inner cavity (see also Fig. 5).

With the gap ratio kept constant at $G=s / R=$ 0.081 , the cross-sectional area of the outer wheelspace is $s \times w \approx 20 \times 28 \mathrm{~mm}^{2}$. While the radial clearance between rotor and stator is fixed at $s_{r}=2 \mathrm{~mm}$, both axial seal clearances at the outer $\left(s_{a, o}\right)$ and inner $\left(s_{a, i}\right)$ location can be adjusted independently in the range between $0-2 \mathrm{~mm}$ $\left(G_{C, \max }=0.0081\right)$ and $0-2.6 \mathrm{~mm}$, respectively. 
Although care was taken in the adjustment of the axial seal clearance, an uncertainty of $0.05 \mathrm{~mm}$ must be acknowledged.

One out of two rotor disks was machined with 93 shank cavities placed just underneath the disk rim. With a rectangular cross-section $(A \approx 8 \times$ $10 \mathrm{~mm}^{2}$ ) and a depth of $20 \mathrm{~mm}$, these elements form a perturbed rotor surface. The according setup is referred to as the 'rough' rotor/plane stator arrangement (setup $A$ ).

During the experiments, air was supplied to the test section at a maximum pressure of 4 atmospheres. The mass flow rate was metered in the range between $0-400 \mathrm{~g} / \mathrm{s}$ by three calibrated metering orifices (uncertainty $\pm 1 \%$ ) and controlled by a bypass throttle valve. Before the air was discharged through the test section, it was settled in an adjacent chamber.

\section{LDV-setup}

The three-dimensional flow field was determined in the outer wheelspace by using a three component fibre-optic LDV-system provided by Dantec. The basic system comprises a 4W Argon-Ion laser in multi mode operation, a standard fibre optics with a $40 \mathrm{MHz}$ Bragg-cell, colour as well as beam separators and two fibre probes. The signals were detected in the back-scattering mode by three photomultipliers in combination with three BSAprocessors. The fibre probes were mounted on a software controlled traverse system and moved within the region of interest with an accuracy of $\pm 2.5 \mu \mathrm{m}$. For every single position, all three velocity components were determined simultaneously on concentric circles. Since the flow pattern relative to the shank cavities was of special interest, the data had to be taken in the absolute frame of reference and transformed into the rotational frame of reference.

Owing to the high centrifugal forces at the rotational speeds investigated and the relatively low strength of available, transparent construction materials, glass or transparent plastics were excluded from the design process of the rotor disk. This circumstance finally led to the radial optical access shown in Figure 2.

The geometrical constraints and the existing flow field aggravated the introduction of seeding particles in such a way that they accumulated onto the inner glass surface. With respect to the signal quality, a contamination of any optical surfaces can not be tolerated. Therefore, compressed air was periodically introduced to clean the critical

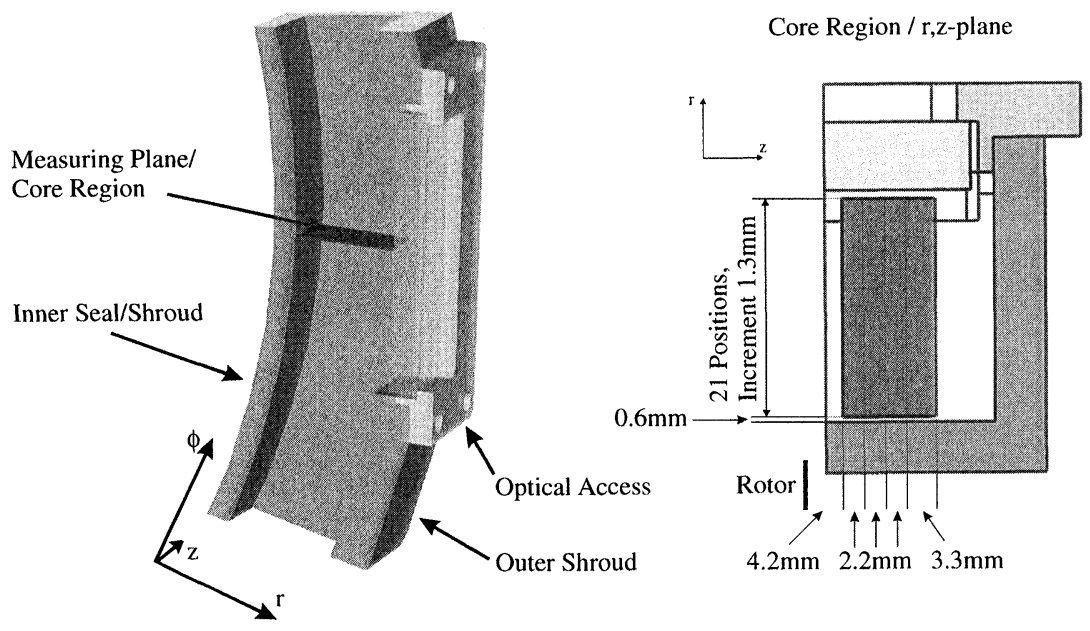

FIGURE 2 Optical access/measuring plane. 
parts within the optical detection path. The data acquisition was paused during clean-up. It should be mentioned that this method worked well in the present setup for solid particles only (Palas DRB5, mean diameter 1-2 $\mu \mathrm{m})$. Small seeding droplets $(d \approx 0.5 \mu \mathrm{m})$ created a dense film on the window which could not be removed during the experiments.

Despite the excellence of modern BSA's, a high $S / N$-ratio must be attained during the experiments. If excessive noise levels can be avoided, high-quality LDV-measurements can be achieved. Due to the large velocity bandwidth in the present investigation, the noise level can increase to the signal's order of magnitude. Additionally, the signal intensity levels are low compared to a configuration, where scattered light would be detected in the forward-scattering mode. In particular for the case where light is reflected from walls or scattered from debris on the window surfaces, the noise level can exceed the signal intensity. Increasing the laser power would help to overcome the problems associated with a polluted optical detection path but would further intensify the reflections.

If LDV-measurements are performed adjacent to walls, scattered light from surrounding surfaces becomes the limiting factor. In the present setup, the measuring plane is surrounded by the inner and outer shroud/lip seal as well as the rotor disk and the stator. In a standard back-scatter setup the probe volume can be several millimeters long leading to severe reflections onto the photomultiplier-tubes when approaching the walls. Therefore, near-wall measurements as shown in Figure 3 are almost impossible even if the walls are coated with a highly absorbing black paint. While the standard setup turned out to fail in this situation, some simple modifications allowed measurements much closer to the walls.

A schematic diagram of the optical path is depicted in Figure 3 for both LDV-probes. To meet the demands of simultaneous, three-dimensional velocity measurements, the foci of both

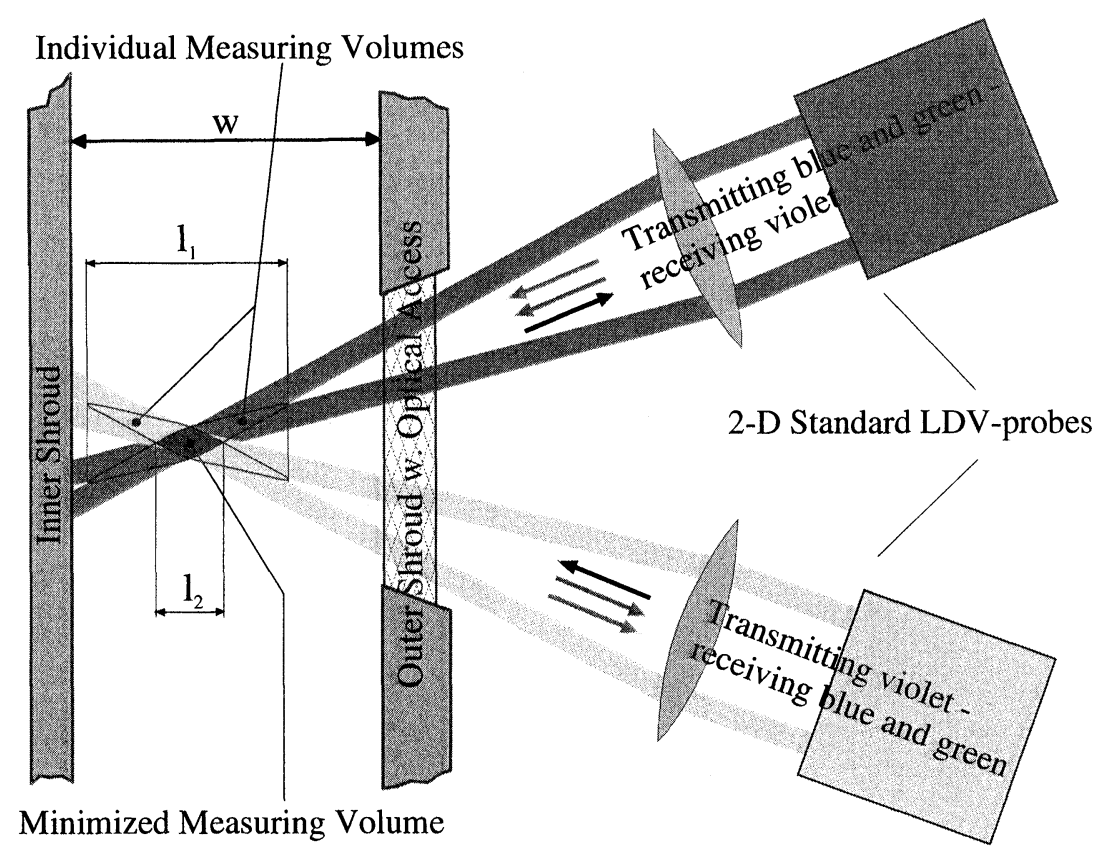

FIGURE 3 Optical detection path. 
lenses must coincide in one single measuring volume. Foci $(f=400 \mathrm{~mm})$ and beam separation $(b=38 \mathrm{~mm})$ of the probes were chosen to cover most of the outer cavity region. These parameters determine the length of the effective measuring volume which is several millimeters in the standard setup. While the geometrical boundary conditions are fixed, a reduction of the measuring volume can be achieved only by changing the detection paths of both probes. In the modified setup, probel is used as transmitter while probe 2 serves as receiver for probe1 and vice versa. As a result, scattered light is detected within a small fraction of the original measuring volume. Its new length is determined by the inclination angle of both probes. At an angle of $78^{\circ}$, the measuring volume is considerably shorter than the original $\left(l_{2} \approx 250 \mu \mathrm{m}\right)$. As a result, flow velocities can be acquired at $1 \mathrm{~mm}$ from the inner wall without excessive reflections. Furthermore, coincidence is ensured by the adjustment of the optics. Therefore, good quality measurements are accomplished at the desired high resolution.

\section{Determination of Moment Coefficients}

The determination of $c_{M}$ was accomplished by means of a precision torque meter, calibrated to an uncertainty of $\pm 0.1 \%\left(M_{\max }=10 \mathrm{Nm}\right)$. The device was placed between the electrical motor and the test section (see Fig. 4). Its axis was aligned to the rig's centerline and mechanical vibrations were minimised by two flexible clutches. The whole apparatus was driven through a flat belt on pulleys.

In the present setup, the experimental results had to be corrected for the frictional torque generated by the bearings. With the rotor disk taken from the drive shaft, the torque was measured as a function of the rotational speed and the axial load. The axial force resulting from the pressure level inside the wheelspace was adjusted by means of a special spring arrangement attached to the shaft. At maximum $c_{w}$, i.e., at the highest axial loads acting on the bearings, the remaining error in $c_{M}$ was determined to be within $\pm 3.1 \%$ (max disk speed) and $\pm 4.9 \%$ (min disk speed). At low mass flow rates the error is approximately $\pm 2.4 \%$.

Thermocouples and pressure taps were located at various positions inside the outer and inner wheelspace to determine the required air properties inside the test section. Figure 5 depicts where static temperatures and static pressures were measured. The uncertainty of $c_{w}$ is $\pm 2 \%$, the error in $\operatorname{Re}_{\varphi}$ was determined to be $\pm 2.4 \%$.

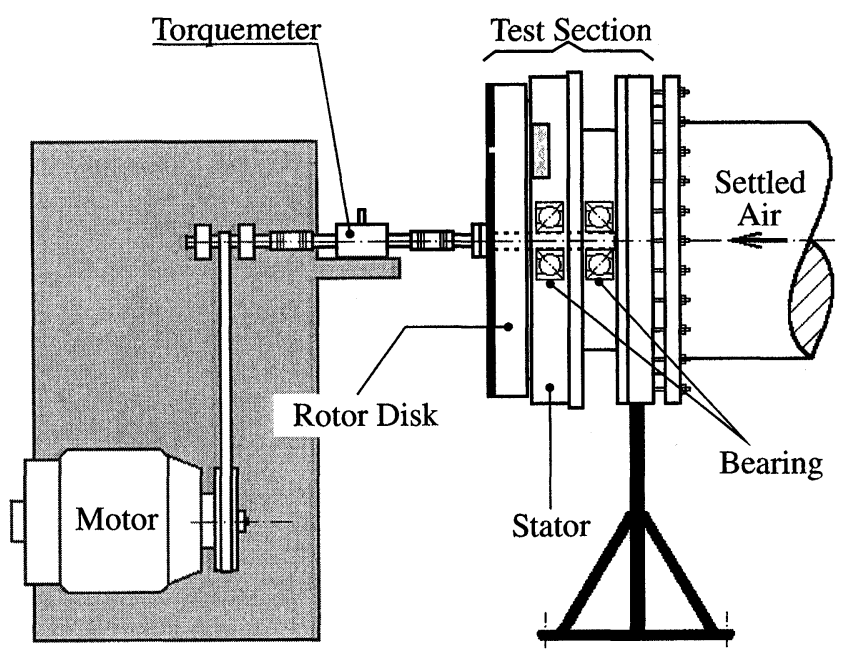

FIGURE 4 Experimental rig. 


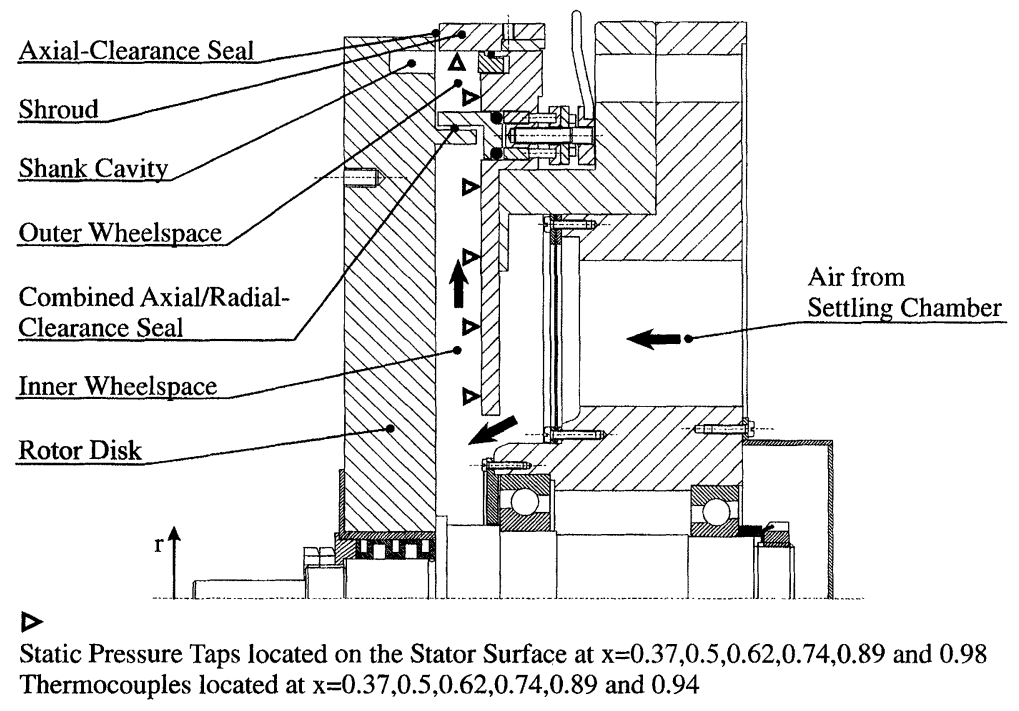

FIGURE 5 Test section.

\section{EXPERIMENTAL RESULTS AND DISCUSSION}

\section{Velocity Measurements}

The three-dimensional, phase-resolved flow field was determined for two rotor-stator combinations. Since it was the goal to investigate the influence of small cavities located inside the rotor wall in the vicinity of the rim seal, the measurements were focussed on the rotor disk with shank cavities (setup $A$ ). The data was finally compared to a plane rotor/ plane stator combination (setup $B$ ).

For both test cases the outer and inner seal clearances were kept constant at $s_{a, o}=1 \mathrm{~mm}$ $\left(G_{c, o}=0.004\right)$ and $s_{a, i}=1.8 \mathrm{~mm} \quad\left(G_{c, i}=0.0073\right)$, respectively. The rotational Reynolds number was fixed at $\operatorname{Re}_{\varphi}=2.5^{*} 10^{6}$, the nondimensional flow rate at $c_{w}=2.2^{*} 10^{4}$. With respect to gas ingestion, the wheelspace is properly sealed under these conditions.

Due to the geometrical constraints, measurements close to the rotor and stator walls required an individual readjustment of the LDV-probes. Separate measurements were carried out in three subdivided areas of the outer wheelspace. Inside the core region (' 1 '), the measuring volume was positioned at 5 different axial locations and moved to 21 radial positions, respectively. The increment was $2.2 \mathrm{~mm}$ in axial and $1.3 \mathrm{~mm}$ in radial direction. The minimum distance from the rotor disk was $4.2 \mathrm{~mm}$. More detailed measurements were carried out in the inner seal region ('2') for both rotor/ stator combinations and closer to the rotating cavities (' 3 ') for the 'rough' rotor/plane stator arrangement only. Additional measurements in the shank cavity region were considered to be not necessary in the plane rotor case and therefore, were left out.

The resulting flow field is depicted in Figure 6 for both rotor-stator combinations. In this type of plotting, all phase-resolved data were arithmetically averaged. Although this approach seems to be contrary to the authors' initial plan to show local flow structures induced by the disk cavities, it gives a complete overview of all data points within the $r-z$-plane. Hence, the basic flow pattern inside the outer wheelspace can better be compared to other test cases. Radial and axial velocities are plotted as vectors whereas the circumferential velocities are omitted for clarity. The data are presented in the absolute frame of reference. 

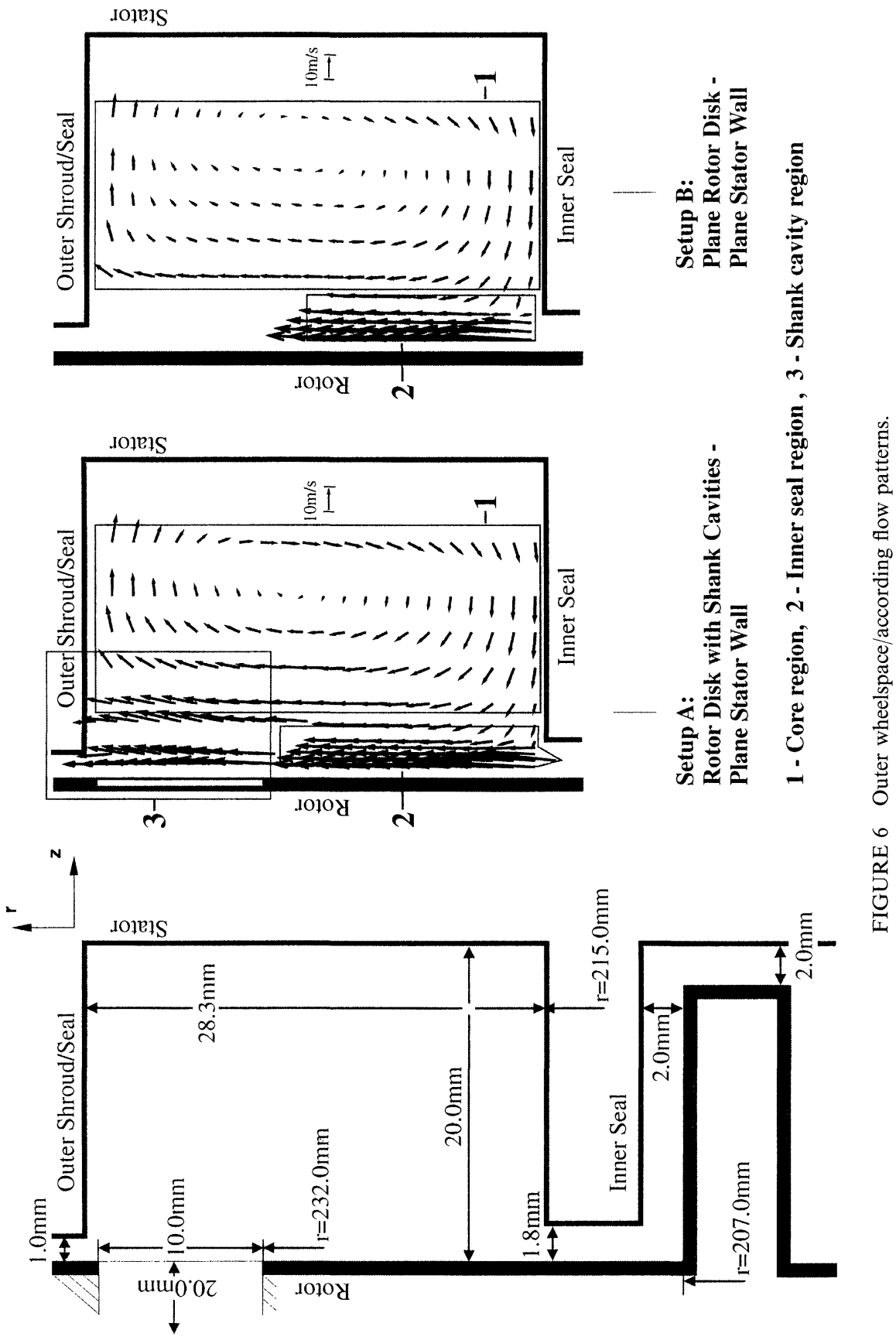
A clockwise toroidal vortex is apparent in both configurations with a central region where radial and axial velocities are minimised. Outside the centre, the flow moves from the rim seal to the stator, radially inward and finally back to the rotor. Employing a flow visualisation technique, this behaviour was earlier reported by Owen and Rogers (1989).

Axial distributions of the tangential velocity are presented in Figure 7. The most conspicuous difference between the two test cases are the higher circumferential velocities in the core region $(0.21 \leq z / s \leq 0.7)$ which are induced by the shank cavities in the rotor wall. In addition, a strong tangential acceleration can be observed for setup $A$ in the vicinity of these elements. Crossing the cavity in radial direction $(\Delta r \approx 10 \mathrm{~mm})$, the flow is tangentially accelerated from $35 \mathrm{~m} / \mathrm{s}$ to $65 \mathrm{~m} / \mathrm{s}$ (see Fig. 9). While most of the air is then discharged through the outer lip seal, some part is redirected at the shroud and contained inside the wheelspace to form the toroidal vortex. Both acceleration and redirection of the flow are responsible for an enhanced transfer of energy into the wheelspace that causes the observed elevated core rotation factors.

At the exit of the combined radial/axial inner seal ('2', Fig. 6), velocities were determined at a higher axial resolution. The left measuring plane is $0.3 \mathrm{~mm}$ distant from the disk surface (setup $A$ ). The increment in axial direction is $0.4 \mathrm{~mm}$, the increment in radial direction $1.3 \mathrm{~mm}$. As can be seen in Figure 8, the disk boundary layer flow is characterised by a maximum circumferential velocity component. At further distance from the rotor $(0.02 \leq z / s \leq 0.05)$, the flow gradually looses momentum due to the enforced interaction with the supplied cooling flow. Discharged through the inner seal, the cooling flow forms some kind of wall jet characterised by a strong radial velocity component. In this region, large tangential velocity gradients can be observed. Axial velocities are close to zero and therefore, are not presented. Moving closer to the stator $(0.08 \leq z / s \leq 0.15)$, the circumferential velocity component increases again owing to the high swirl inside the core. At the interface between the wall jet and the core region, part of the circulating air is entrained into the cooling flow, slowed tangentially and accelerated in radial direction. At the same time, the cooling flow is affected by the entrained air resulting in a higher angular momentum. As a consequence, the tangential velocities near the rotor wall are slightly higher than those observed for the plane rotor case.

In setup $B$, velocities were acquired in the inner seal region at $0.3 \mathrm{~mm}$ distant from the rotor disk. The axial increment is $0.55 \mathrm{~mm}$, the radial increment $1.3 \mathrm{~mm}$. Compared to the configuration with shank cavities in the rotor wall, the tangential velocities decrease steadily with increasing distance from the plane disk surface. The measured values near the rotor wall are slightly smaller than those obtained for the previous test case and therefore, the shear stresses in the disk boundary layer should be slightly larger. However, as will be shown in the next chapter, this circumstance must have little effect on $c_{M}$ compared to the impact of the shank cavities.

Local flow structures in the vicinity of the disk cavities are shown in Figure 10. An angular segment of $31^{\circ}$ is chosen and the data plotted in the $\phi-z$-plane $(r=$ constant $)$. The tangential and axial velocities are presented in the rotational frame of reference as vectors.

At short distance from the rotor disk $(z=0.5-$ $3.1 \mathrm{~mm}$ ), periodic disturbances of the aforementioned wall jet can be observed that are synchronous to the position of the disk shank cavities. In contrast to the authors' initial expectations, there are no vortex structures shed into the external flow which rather seems to 'ride' over the crest of the disk elements. Observed for the non-rotational case, Perry et al. (1969) referred to this type of flow as the ' $d$ ' type rough-wall flow.

Despite the fact that the observed deflection of the flow in positive axial direction is only weak (maximum inclination angle $3.5^{\circ}$ in the rotational frame of reference), the cavities are responsible for an enhanced transfer of momentum into the 


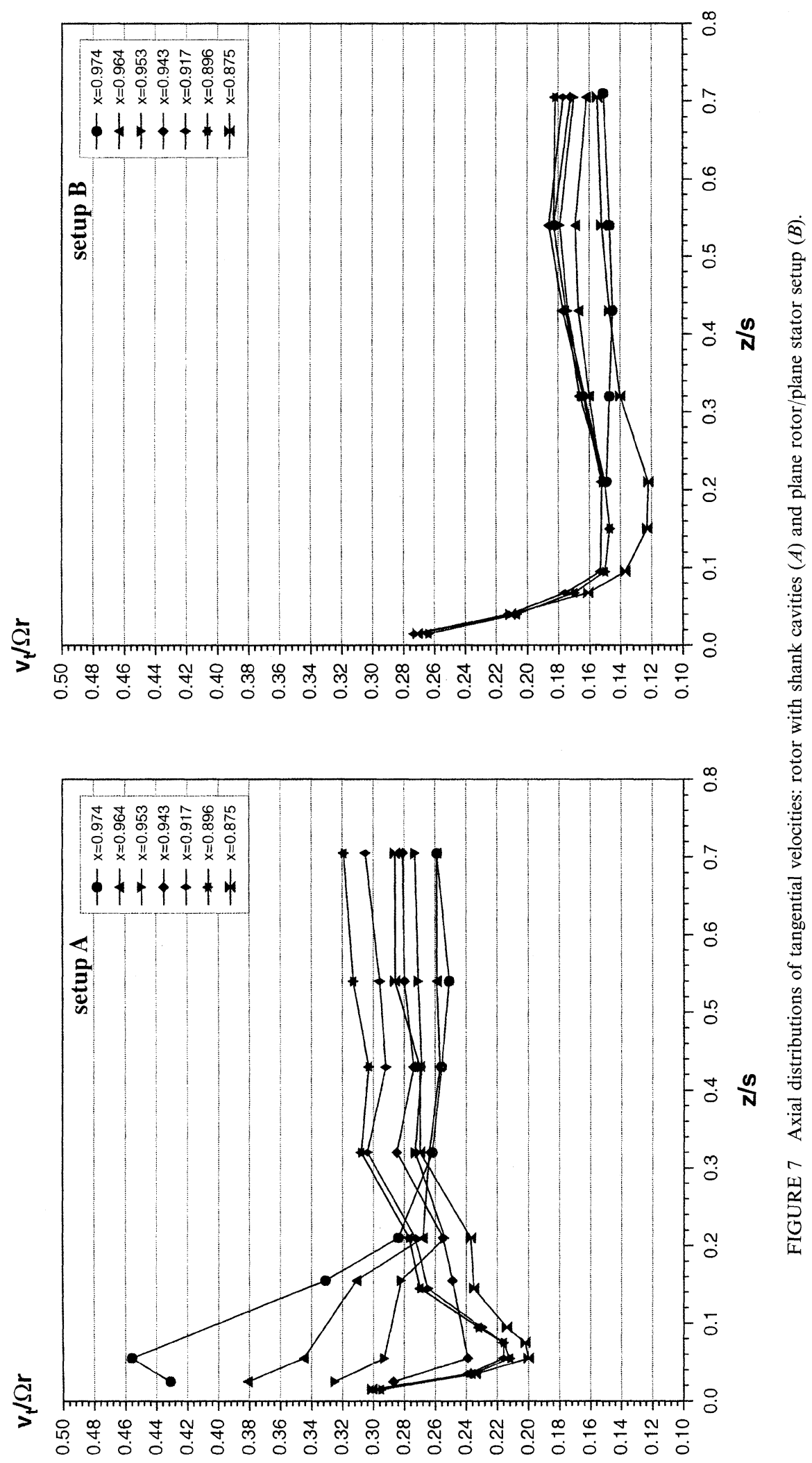



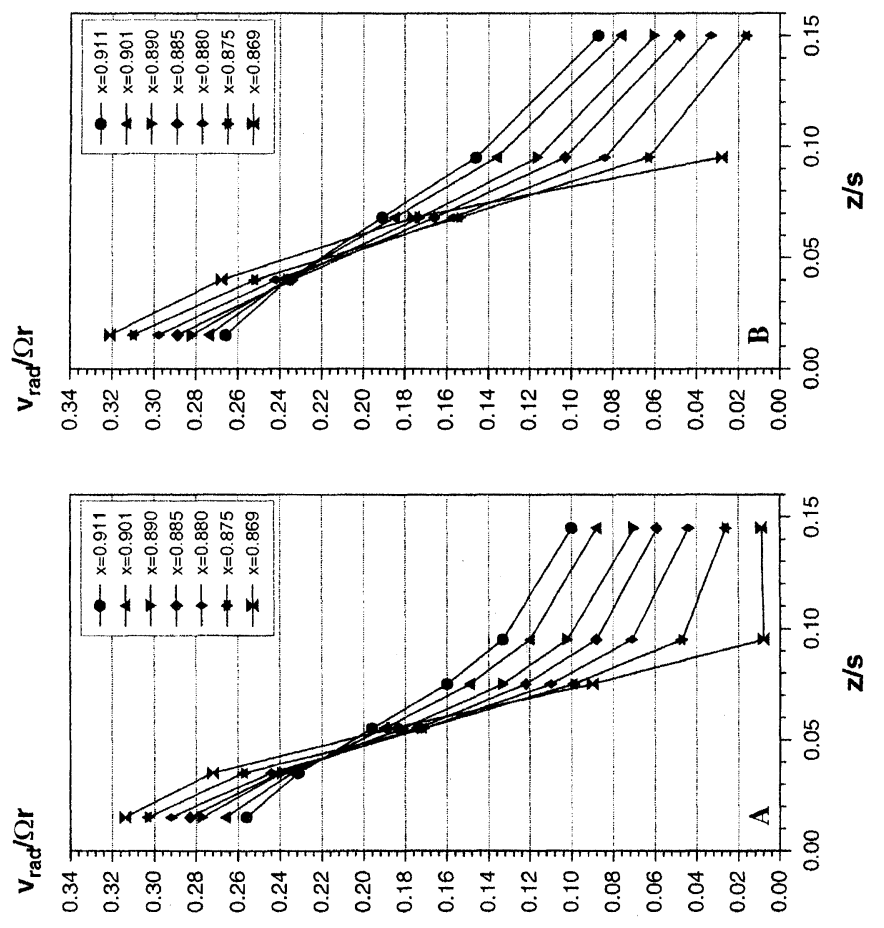

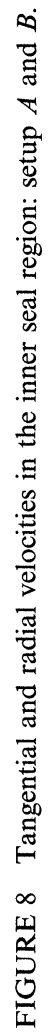

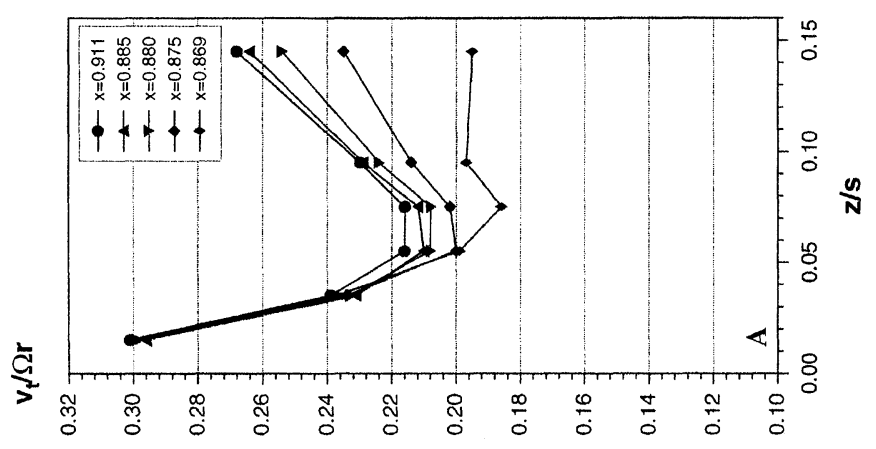




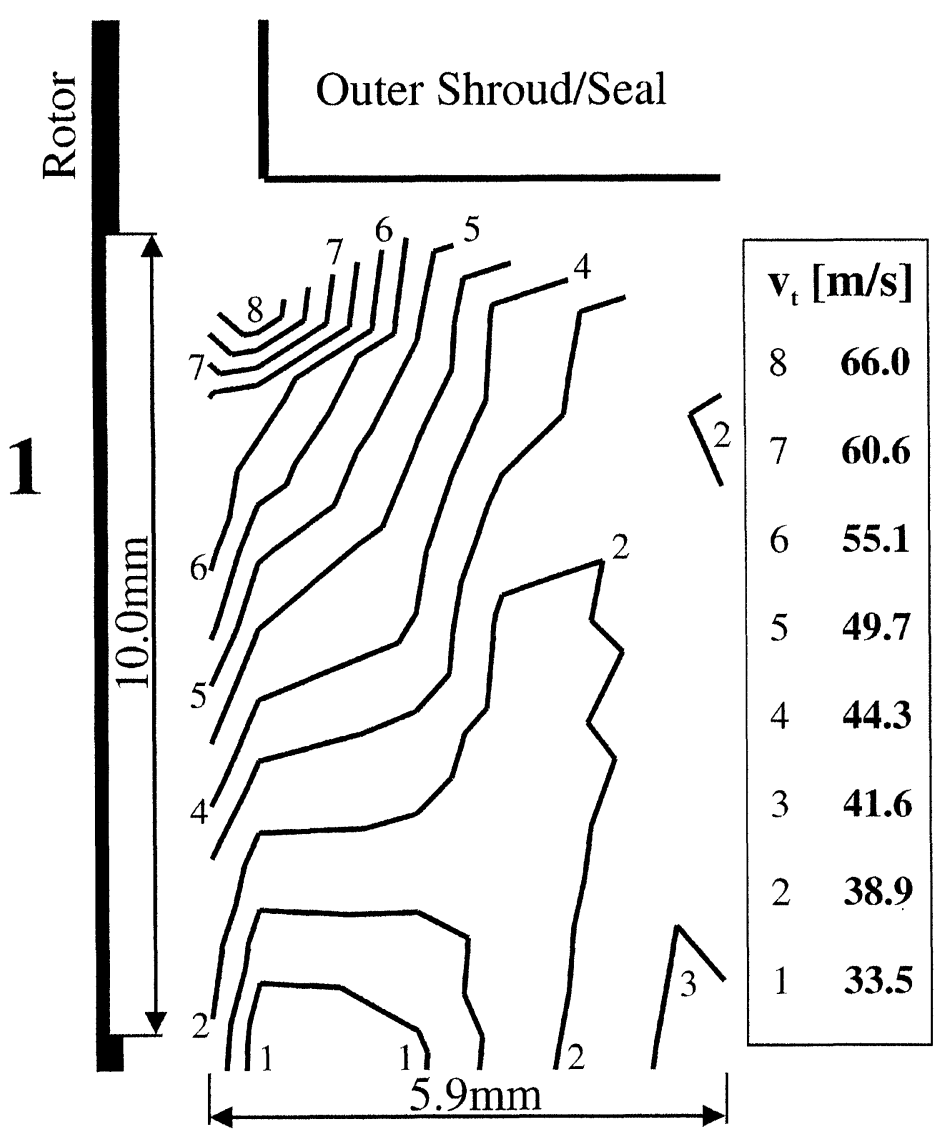

FIGURE 9 Tangential velocities in the shank cavity region.

annulus between the two shrouds. Compared to the case with a plane rotor, this mechanism induces higher shear stresses at the interface between the rotor boundary layer and the main flow as well as higher tangential velocities inside the core region.

\section{Torque Measurements}

The frictional torque was measured for two rotor-stator configurations and various flow parameters. Thereby, the nondimensional moment coefficient $c_{M}$ was computed for the whole disk and correlated with the disk rotational Reynolds number $\operatorname{Re}_{\varphi}$.

The outcome for the plane rotor/plane stator combination is depicted in Figure 11 for several nondimensional flow rates $c_{w}$. Being in qualitative agreement with previous data as reported by Owen and Rogers (1989), $c_{M}$ increases with $c_{w}$ and decreases with $\operatorname{Re}_{\varphi}$. While the behaviour of the system is throughflow dominated for the whole range of parameters, it is rotation dominated in particular for low rotational Reynolds numbers. At increased disk speeds, the influence of both $c_{w}$ and $\operatorname{Re}_{\varphi}$ diminishes.

The absolute increase $\Delta c_{M}$ caused by the shank cavities in the rotor disk is shown in Figure 12. The most predominant characteristic of the plot is the negative slope of the graphs. Starting at moment coefficients $60 \%$ higher compared to the combination with a plane rotor, the effect of the perturbed rotor surface diminishes as the cavity tangential velocity is increased. Recalling the reported mechanisms that are responsible for the increased 


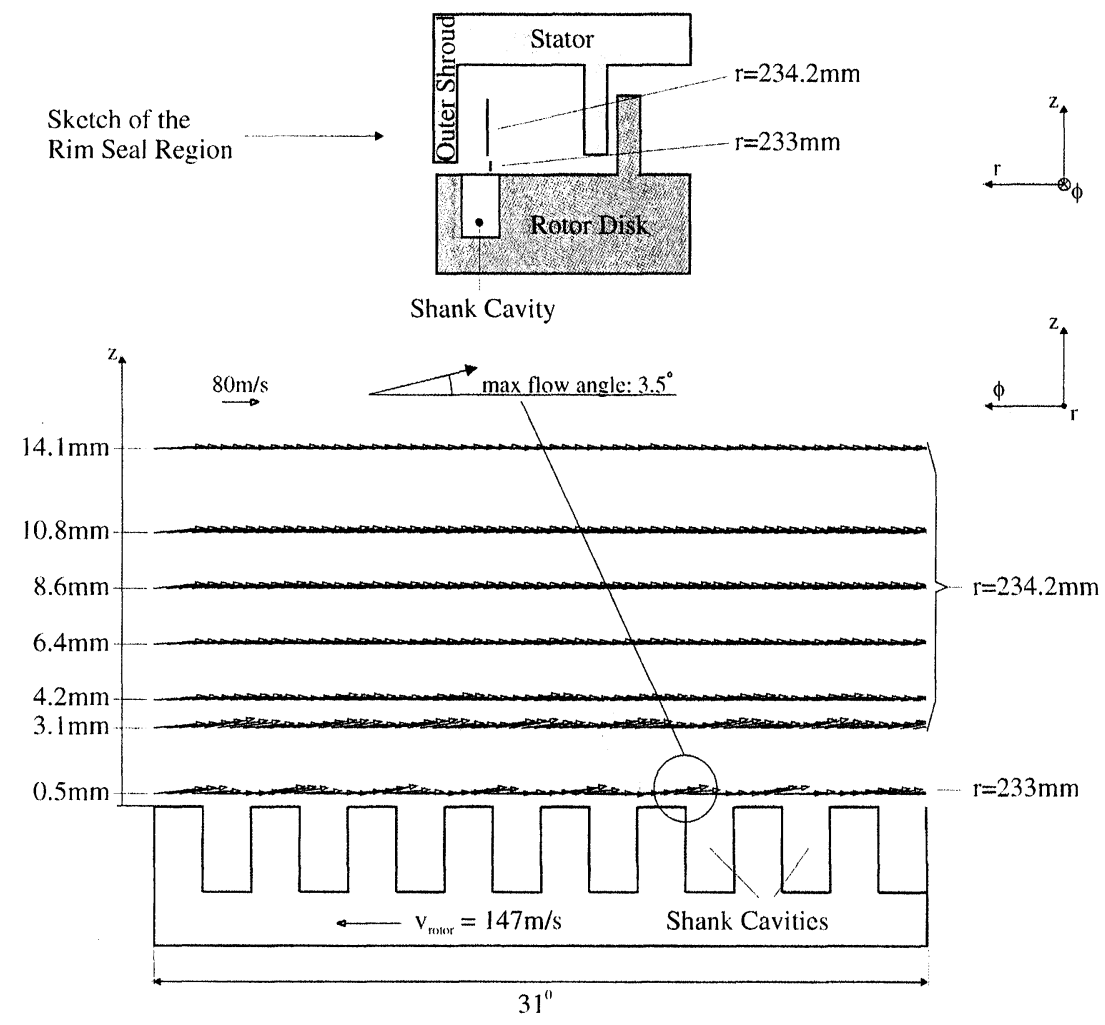

FIGURE 10 Local flow structures.

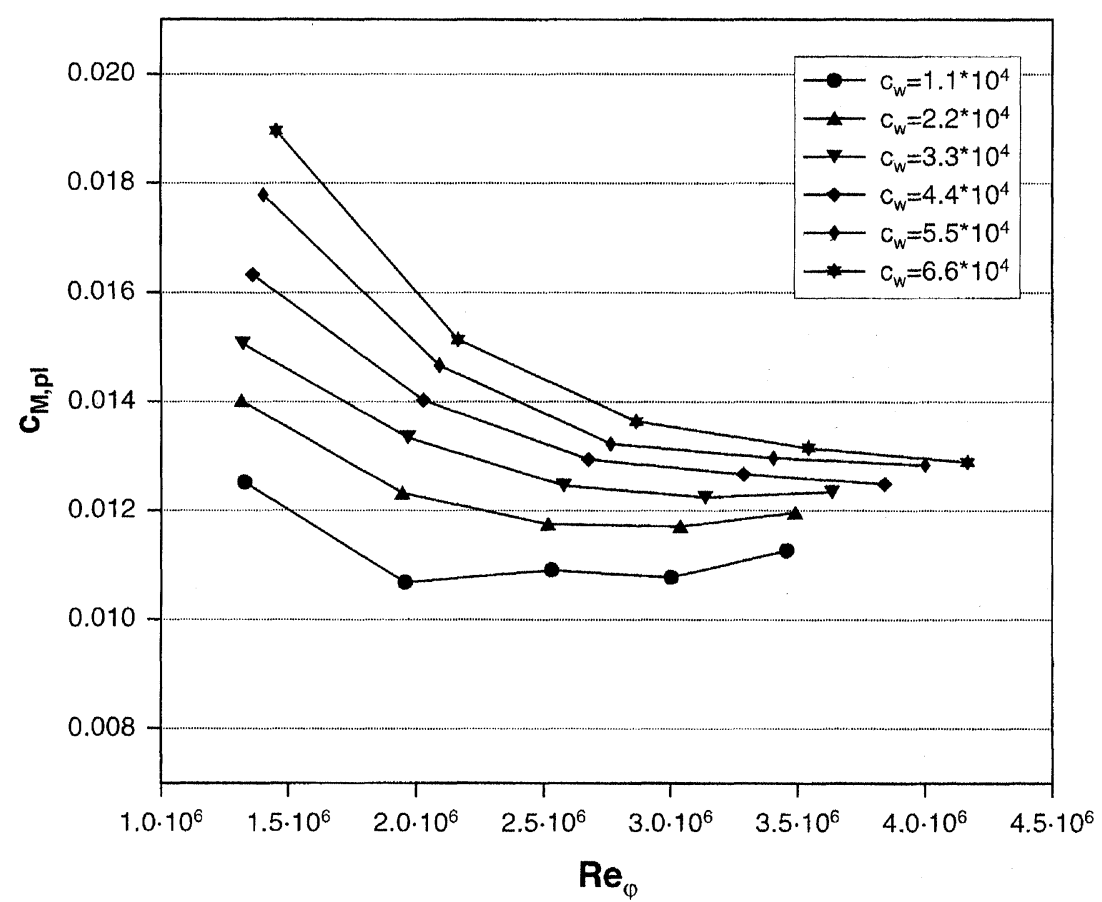

FIGURE 11 Moment coefficients $c_{M}$ for the plane rotor/plane stator configuration (setup $B$ ). 


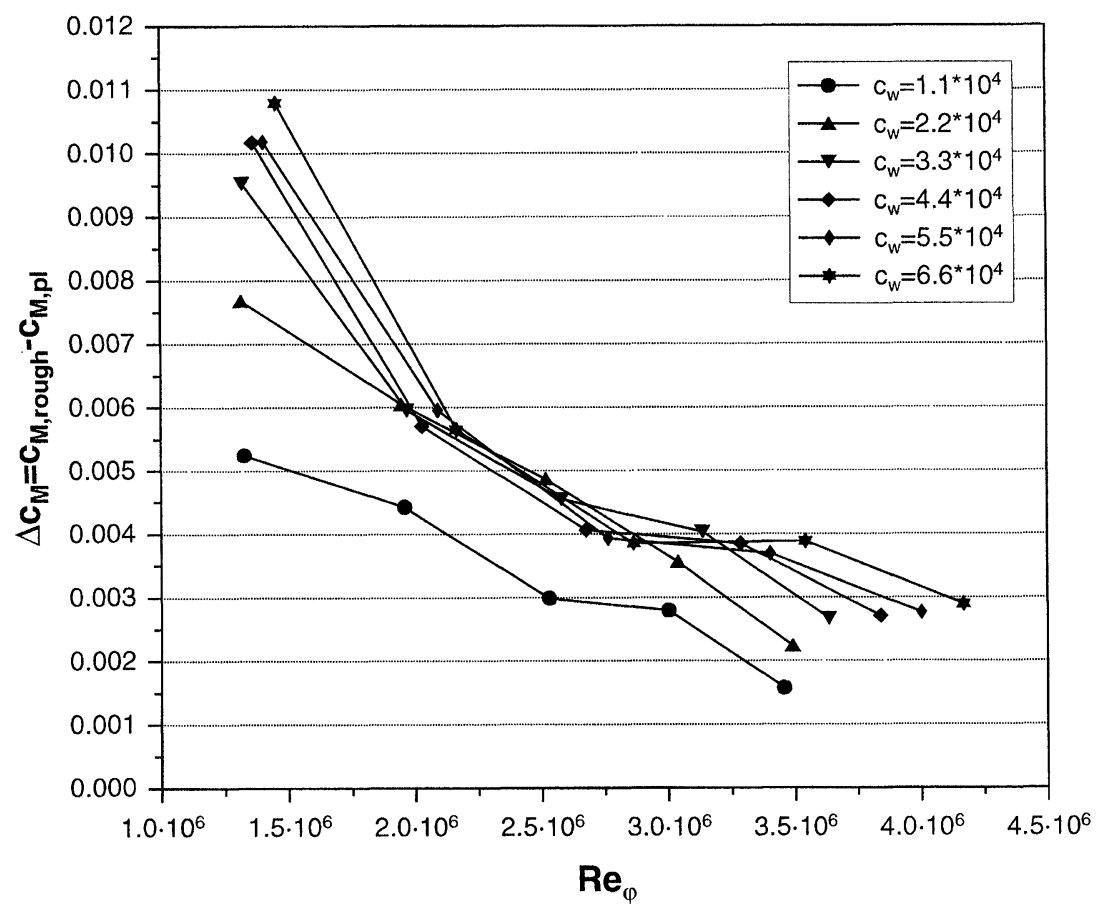

FIGURE 12 Increase in $c_{M}$ for setup $A$.

drag on these elements (Roshko, 1955), one can presume that the deflection of part of the separated boundary layer into the cavity is weaker at high rotational speeds. From the data plotted in Figure 12, it can also be assumed that the influence gradually vanishes if the rotor speed is further raised. With respect to corresponding flow patterns, this is probably the case when the transfer of momentum is blocked by a main flow that overflows the cavities. However, as many authors have reported for the non-rotational case, rounding the cavity edges can severely affect the stagnation pressure at the downstream cavity wall and thus influence the drag force. Since the edges are sharp-edged in the present study, round or chamfered edges still have to be considered in future investigations.

The effect of the flow rate on the absolute increase $\Delta c_{M}$ is not clear in Figure 12. Compared to a simplified test case with a single, stationary cavity in a wind tunnel, the flow field next to a rotating cavity arrangement inside a typical wheelspace is rather complex. While a radial outflow of coolant will change the principal character of the flow pattern (Pincombe, 1989), rectangular cavities or grooves can cause severe and erratic fluctuations (Roshko, 1955 and Kistler and Tan, 1967). Switching flow patterns might be the reason for the fluctuations observed in Figure 12 for the 'rough' rotor/plane stator arrangement. However, from the LDV data presented, there is no such evidence. In contrast to the non-rotational case, the existence of unstable vortex structures inside the cavity is tougher to prove. Owing to the complex flow field, the formation mechanisms are more difficult to study.

Although a proper correlation can not be established in this particular situation, the experiments have clearly shown that a considerable increase of the moment coefficient will be obtained in the presence of shank cavities. At the highest rotational Reynolds numbers investigated, the results are still $20 \%$ higher than those obtained for the plane rotor. With regard to the relative increase, it must be noted that $c_{M}$ is specified for both sides of the disk. 


\section{CONCLUSIONS}

The three-dimensional, phase resolved flow field was obtained in the rim seal cavity of a doubleshrouded rotor-stator system. Special interest was directed towards small rectangular cavities located at the circumference of the rotor disk. Besides local flow structures in the vicinity of these elements, moment coefficients are also presented.

With the surface irregularities formed by the cavities, circumferential velocities were measured in the core region of the outer wheelspace that are higher than those obtained for the setup with a plane rotor. Elevated tangential velocities indicate that there is an enhanced transfer of momentum from the cavities to the main flow. This mechanism is confirmed by the torque measurements. Higher moment coefficients were determined for the whole range of flow parameters.

The flow pattern close to the disk surface is relatively undisturbed by the perturbations in the rotor wall. It is similar to that described by Perry et al. (1969) for the non-rotational case. These authors referred to this type of flow as the ' $d$ '-type roughness flow.

The relative increase of the moment coefficient due to the shank cavities decreases with the disk rotational Reynolds number. For the range of parameters investigated, values between $20 \%$ and $60 \%$ were obtained. The influence of $c_{w}$ is sketchy for medium Reynolds numbers. The fluctuations observed are possibly of the same origin as those reported by Roshko (1955) and Kistler and Tan (1967). Owing to the measuring techniques employed in the present investigation, neither pressure nor velocity distributions could be acquired inside the rectangular cutouts that could backup the occurrence of non-stationary flow phenomena.

From the outcome of this work it can be concluded that windage power will be increased by the existence of the disk shank cavities due to the increased shear stresses in the disk and stator boundary layers. While power losses caused by the elements deteriorate the turbine efficiency to a certain degree, the assumed transport into and out of the cavity might also be reflected by an improved heat transfer from the rotor surface. The latter subject is to be considered in future investigations.

The present work is incomplete in a sense that only a small fraction of all possible flow cases or geometry variations have been considered. However, the authors think that the velocity data presented reveal the basic mechanisms that cause the observed rise of $c_{M}$. In order to gain a more detailed understanding of the fluctuations observed for the perturbed rotor, additional effort is required.

\section{Acknowledgements}

The authors gratefully acknowledge support for this work by the German BMWi. The experimental work presented here was part of the German Luftfahrtforschungsprogramm 19951998. Special thanks are due to Dr. W. Waschka for the coordination of the programme.

\section{NOMENCLATURE}

\begin{tabular}{|c|c|}
\hline$A$ & Area $\left[\mathrm{m}^{2}\right]$ \\
\hline$b$ & Beam separation $[\mathrm{m}]$ \\
\hline$c_{M}$ & $\begin{array}{l}\text { Moment coefficient for the rotor disk } \\
\left(=M /(1 / 2) \rho \Omega^{2} R^{5}\right)[-]\end{array}$ \\
\hline$c_{w}$ & $\begin{array}{l}\text { Nondimensional flow rate }\left(c_{w}=\dot{m} / \mu \cdot R\right) \\
{[-]}\end{array}$ \\
\hline$d$ & Droplet diameter $[\mathrm{m}]$ \\
\hline$f$ & Focus length $[\mathrm{m}]$ \\
\hline$G$ & Gap ratio $(G=s / R)[-]$ \\
\hline$G_{c}$ & Seal clearance ratio $\left(G_{c}=s_{a, o} / R\right)[-]$ \\
\hline$l$ & Length of measuring volume [m] \\
\hline$M$ & Moment for the rotor disk $[\mathrm{Nm}]$ \\
\hline$\dot{m}$ & Mass flow rate of sealing air $[\mathrm{kg} / \mathrm{s}]$ \\
\hline$n$ & Rotational speed of rotor disk $[1 / \mathrm{min}]$ \\
\hline$r$ & Radius [m] \\
\hline$R$ & Outer radius of rotor disk [m] \\
\hline $\operatorname{Re}_{\varphi}$ & $\begin{array}{l}\text { Rotational Reynolds number }\left(=\rho \Omega R^{2} / \mu\right) \\
{[-]}\end{array}$ \\
\hline$S$ & Axial gap between rotor and stator $[\mathrm{m}]$ \\
\hline
\end{tabular}


$s_{a, i} \quad$ Inner seal axial clearance $[\mathrm{m}]$

$s_{a . o} \quad$ Outer seal axial clearance [m]

$s_{r} \quad$ Inner Seal radial clearance [m]

$v_{\text {rad }} \quad$ Radial velocity $[\mathrm{m} / \mathrm{s}]$

$v_{t} \quad$ Tangential velocity $[\mathrm{m} / \mathrm{s}]$

$w \quad$ Width of rim seal cavity [m]

$x \quad$ Nondimensional radius $(=r / R)[-]$

$z \quad$ Axial coordinate measured from disk [m]

$\Delta \quad$ Difference [-]

$\phi \quad$ Angular coordinate [-]

$\mu \quad$ Dynamic viscosity [Pas]

$\nu \quad$ Kinematic viscosity $\left[\mathrm{m}^{2} / \mathrm{s}\right]$

$\rho \quad$ Density $\left[\mathrm{kg} / \mathrm{m}^{3}\right]$

$\Omega \quad$ Angular speed of the rotor $(=2 \pi n / 60)$ $[1 / \mathrm{s}]$

\section{Subscripts}

$\max$ Maximum

rough Rotor disk with shank cavities

pl Plane rotor

\section{References}

Gärtner, W. (1998) A Momentum Integral Method to Predict the Frictional Torque of a Rotating Disk with Protruding Bolts, ASME paper 98-GT-138.

Geis, T., Wiebelt, A., Kim, S. and Wittig, S. (1999) Ingress Determination by means of Laser Light Scattering inside a Rotor-Stator System, ASME paper 99-GT-247.

Haugen, R. L. and Dhanak, A. M. (1966) Momentum Transfer in Turbulent Separated Flow Past a Rectangular Cavity, Trans, ASME Journal of Applied Mechanics, pp. 641-646.

Kistler, A. L. and Tan, F. C. (1967) Some Properties of Turbulent Separated Flows, The Physics of Fluids, 10(9)(II), $165-173$.

Nece, R. E. and Daily, J. W. (1960) Roughness Effects on Frictional Resistance of Enclosed Rotating Disks, Journal of Basic Engineering, pp. 553-561.

Owen, J. M. and Rogers, R. H. (1989) Flow and heat transfer in rotating disc systems, Vol. 1-Rotor-Stator-Systems, Research Studies Press, Taunton, U.K.; Wiley, New York.

Pincombe, J. R. (1989) Gas Turbine Disk Cooling Flows, In: Handbook of Flow Visualization, Hemisphere Publishing Corporation, pp. 555-576.

Perry, A. E., Schofield, W. H. and Joubert, P. N. (1969) Rough wall turbulent boundary layers, Journal of Fluid Mechanics, 37(2), 383-413.

Roshko, A. (1955) Some Measurements of Flow in a Rectangular Cutout, NACA TN 3488.

Zimmerman, H., Firsching, A., Dibelius, G. H. and Ziemann, M. (1986) Friction Losses and Flow Distribution for Rotating Disks With Shielded and Protruding Bolts, ASME paper 86-GT-15. 

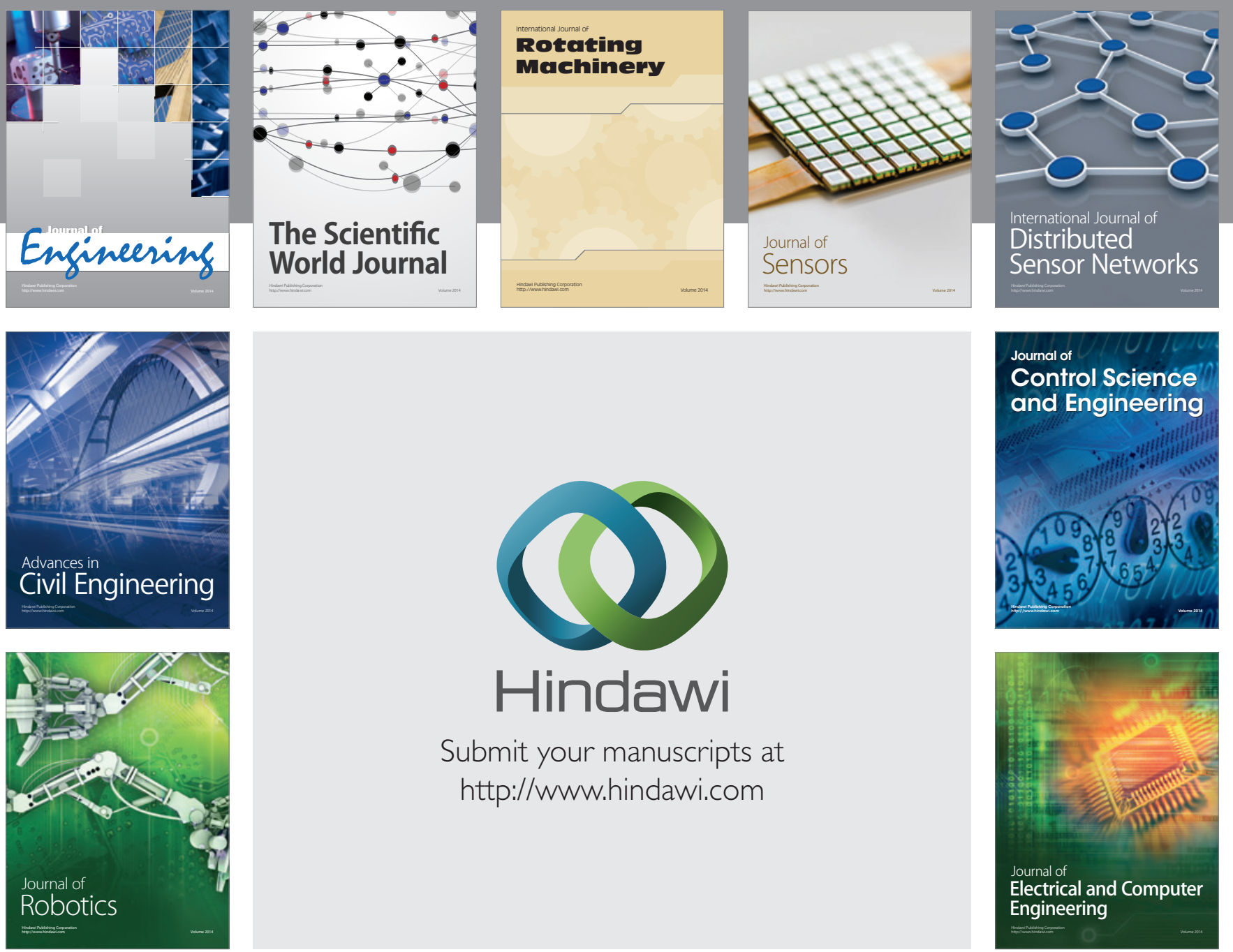

Submit your manuscripts at

http://www.hindawi.com
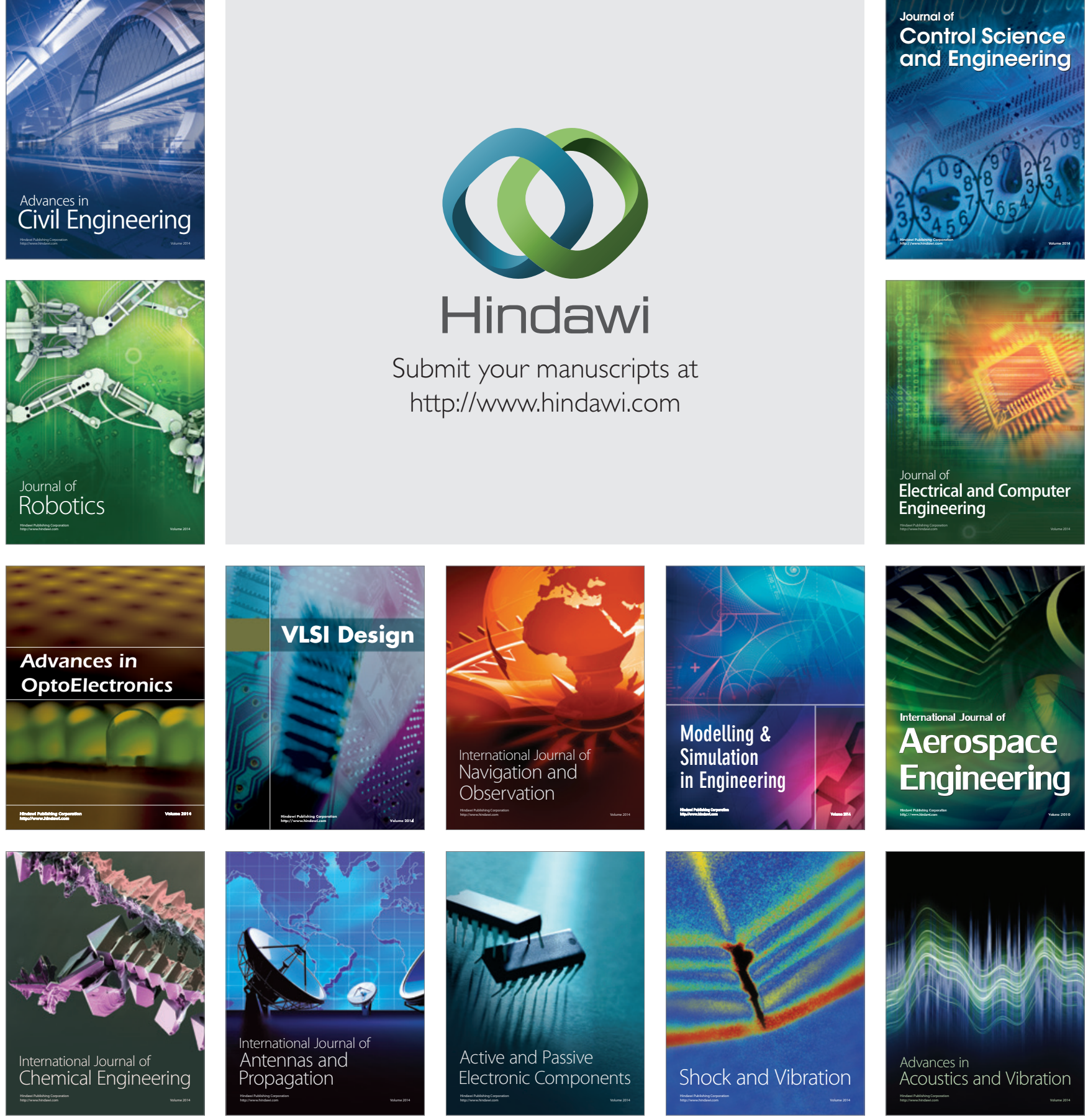